\title{
Effect of azithromycin on enhancement of methane production from waste activated sludge
}

\begin{abstract}
In the methane production from waste activated sludge (WAS), complex bacterial interactions in WAS have been known as a major contribution to methane production. Therefore, the influence of bacterial community changes toward methane production from WAS was investigated by an application of antibiotics as a simple means for it. In this study, azithromycin (Azm) as an antibiotic was mainly used to observe the effect on microbial changes that influence methane production from WAS. The results showed that at the end of fermentation, Azm enhanced methane production about twofold compared to control. Azm fostered the growth of acid-producing bacterial communities, which synthesized more precursors for methane formation. DGGE result showed that the hydrolysis as well as acetogenesis stage was improved by the dominant of B1, B2 and B3 strains, which are Clostridium species. In the presence of Azm, the total population of archaeal group was increased, resulting in higher methane productivity achievement.
\end{abstract}

Keyword: Azithromycin; Bacterial community; Methane production; Sludge hydrolysis; Waste-activated sludge 